\title{
AFM characterization of the shape of surface structures with localization factor
}

\begin{abstract}
Attila Bonyár
Department of Electronics Technology, Budapest University of Technology and Economics, Egry József Street 18, Budapest, H-1111, Hungary bonyar@ett.bme.hu
\end{abstract}

Keywords: localization factor, structural entropy, AFM, surface roughness

\begin{abstract}
Although with the use of scanning probe microscopy (SPM) methods the topographical imaging of surfaces is now widely available, the characterization of surface structures, especially their shape, and the processes which change these features is not trivial with the existing surface describing parameters. In this work the application of a parameter called localization factor is demonstrated for the quantitative characterization of surface structures and for processes which alter the shape of these structures. The theory and optimal operation range of this parameter is discussed with three application examples: microstructure characterization of gold thin films, characterization of the changes in the grain structure of these films during thermal annealing, and finally, characterization of the oxidation processes on a polished tin surface.
\end{abstract}

\section{Introduction}

In the field of surface science the purpose of atomic force microscopy (AFM) is usually besides imaging - the numerical characterization of the surface topography in order to correlate the microscopic, or even nano-level features to the macroscopic properties or behavior of the material in a quantitative way. For this purpose the ISO 25178-2 standard offers a total number of 30 parameters for 3D areal surface characterization. These parameters are categorized in five groups: height parameters, spatial parameters, hybrid parameters, functional parameters and parameters related to segmentation. In practice the height parameters (e.g. the surface roughness) are the most commonly used, although they are simple average based values. For example classical surface roughness parameters obtained with AFM are frequently used as a typical measure of mechanical surface properties due to its influence on e. g. wettability (Xia et al., 2016), friction and wear (Rahaman et al., 2015), thermal conductance and radiation (Chen et al., 2015) etc. There are also lots of medical and bioengineering applications were the surface roughness is commonly used, e.g. for the characterization of orthodontic components (Lee et al., 2010) or for intraocular lenses (Mukherjee et al, 2012). In the case of medical (mainly dental) implants there is a close correlation between roughness and interfacial shear strength (Hansson et al., 2011).

From a critical point of view we can have the following reservations regarding these frequently used height parameters: 1) usually one parameter is not enough to identically characterize a surface (it can be very easily proven that surfaces with completely different structures can have the same average surface roughness); 2) there is no parameter in the ISO standard which directly characterizes the shape of the surface structures (although there are parameters, which indirectly 
carry information regarding the shape); 3) the simple, average based parameters, which are calculated for the whole image are very sensitive to artifacts, noise and generally to the whole post processing of the image (e.g. in the case of surfaces with high aspect ratio, the background correction of the AFM images is not trivial (Molnár et al., 2010)).

In 2012 a novel parameter called localization factor was introduced for the evaluation of AFM images, especially to characterize the shape of the structures under investigation (Bonyár et al., 2012). Since its introduction it was also proven that this parameter - as it will be discussed later is also less sensitive to imaging artifacts and post processing (Bonyár et al., 2013).

The current work has two major aims. First, by defining the operation range of localization factor - the range and combination of imaging parameters (e.g. scan size, resolution) and calculation parameters (e.g. segmentation number) with relation to the structures to be characterized, with which the calculation of the localization factor is stable - a guidance is given to be able to obtain reliable and meaningful localization factor values in every application. Secondly, three application examples are given to demonstrate the use of this parameter for 1) the characterization of technologically different gold thin film microstructures (Section 4.1); 2) the characterization of the changes in the shape of the grains observed during thermal annealing of such gold thin films (Section 4.2); 3) for the characterization of tin surface oxidation (Section 4.3). Besides the presented examples other successful applications of this parameter can be found in the literature, e.g. for the characterization of heat flux in two-dimensional solids (Wang et al., 2015).

\section{Theory}

In this chapter the theoretical background of the most relevant ISO parameters - concerning the application areas to be presented - is discussed (Section 2.1) along with the background and calculation method of the localization factor (Section 2.2). Finally, the considerations behind the determination of the operation range of this parameter is discussed (Section 2.3).

\subsection{Relevant ISO parameters}

AFM topography images can be interpreted as a distribution of pixel intensities where a height value $\left\{z_{i}, i=1, \ldots, N\right\}$ is assigned to each point of the scan range $\left\{\left(x_{i} ; y_{j}\right), i=1, \ldots, M, j=1, \ldots, M\right\}$ which is proportional to the pixel intensity $\left\{I_{i}, i=1, \ldots, N\right\}$, where $M$ is the number of scan points per row/column (resolution) and $N$ is the total number of scan points $(N=M \times M)$. The simplest numerical parameters which could be defined on the surface include the maximum peak height, $\left(S_{p}\right)$, the maximum valley depth, $\left(S_{v}\right)$ and their sum, the maximum height, $\left(S_{z}\right)$.

There are statistical parameters to describe the shape of the height distribution function (HDF). The most widespread are the surface roughness $\left(S_{a}\right)$ and the RMS (root mean square) surface roughness $\left(S_{q}\right)$, which could be defined as the following:

$S_{a}=\frac{1}{N} \sum_{i=1}^{N}\left|\eta_{i}\right|$ 
$S_{q}=\sqrt{\frac{1}{N} \sum_{i=1}^{N} \eta_{i}^{2}}=\mu_{2}^{1 / 2}$

In the definition of these parameters $\eta_{i}$ is the distance between a height point $\left(z_{i}\right)$ and the central plane defined for the surface. $\mu_{2}$ is the second central moment of the distribution. The k-th central moment can be defined as (3), where $\rho(z)$ is the probability density function of the height distribution.

$\mu_{k}=\int_{-\infty}^{\infty} \eta_{i}^{k} \rho(z) \mathrm{d} z$

Based on the higher moments of the probability distribution we can define the skewness $\left(S_{s k}\right)$ and the kurtosis $\left(S_{k u}\right)$.

$S_{s k}=\frac{\frac{1}{N} \sum_{i=1}^{N} \eta_{i}{ }^{3}}{\left(\frac{1}{N} \sum_{i=1}^{N} \eta_{i}{ }^{2}\right)^{3 / 2}}=\frac{\mu_{3}}{\mu_{2}^{3 / 2}}$

$S_{k u}=\frac{\frac{1}{N} \sum_{i=1}^{N} \eta_{i}{ }^{4}}{\left(\frac{1}{N} \sum_{i=1}^{N} \eta_{i}{ }^{2}\right)^{2}}-3=\frac{\mu_{4}}{\mu_{2}^{2}}-3$

These four parameters are often categorized as height (or amplitude) parameters in the ISO standard. Besides the HDF (which is a first order distribution) we can use second order distributions for the characterization of the AFM images as well. These so called spatial parameters characterize the spatial periodicity of the surface and its direction. The most well know is the autocorrelation function $(\mathrm{ACF})$ and the autocorrelation length $\left(S_{a l}\right)$, which is derived from this distribution (see for example Nečas et al., 2013). If the surface has a directed texture, the autocorrelation length characterize the fastest decay in the direction which is perpendicular to the texture. The texture aspect ratio $\left(S_{t r}\right)$ is a ratio between the fastest and slowest decay lengths, and characterize the homogeneity of the surface. For a completely isotropic surface $S_{t r}=1$, for a texture with direction (anisotropy) $S_{t r}=0$. In the case of an anisotropic surface the texture direction $S_{t d}$ gives the angle between the texture direction and the scanning direction in degrees.

Taking into consideration the purpose of our investigations we think, that two more ISO parameters could be relevant regarding the description of the grain's shape. These two are categorized as hybrid parameters and are related to the spatial shape of the data. The RMS gradient of the surface $\left(S_{d q}\right)$ characterizes the slopes on the surface.

$S_{d q}=\sqrt{\frac{1}{(N-1)^{2}} \sum_{l=1}^{N} \sum_{k=1}^{N}\left(\frac{\partial \eta\left(x_{k}, y_{l}\right)}{\partial x}\right)^{2}+\left(\frac{\partial \eta\left(x_{k}, y_{l}\right)}{\partial y}\right)^{2}}$

The developed area ratio $\left(S_{d r}\right)$ is the ratio between the real surface area $\left(A_{r}\right)$ and the geometrical area $(A)$, where the latter is equal to the scan size.

$S_{d r}=\frac{A_{r}}{A}$ 
For the detailed description and application of the 3D surface parameters in general we recommend for example the work of Raposo et al. (2007).

\subsection{Localization factor}

In this section we discuss the necessary mathematical background to understand the localization factor parameter. For the detailed description of this parameter see the original article regarding its introduction for the characterization of thin film surfaces (Bonyár et al, 2012).

To calculate the localization factor one must calculate the generalized localization of an AFM image. The generalized localization can be derived from the structural entropy $\left(S_{s t r}\right)$ and the spatial filling factor $(q)$ functions of the image. Since the intensity distribution $\left(I_{i}\right)$ of a contact-AFM image can be normalized in a way to fulfill equations (8) and (9), the image can be well characterized with the structural entropy and the spatial filling factor.

$Q_{i} \geq 0$ for $i=1, \ldots, N$

$\sum_{i=1}^{N} Q_{i}=1$

The spatial filling factor is derived from the participation ratio (or delocalization measure, $D$ ) which is a well-known quantity in density matrix analysis, introduced by Bell and Dean (1970) and Pipek (1989) independently. The definition modified to the case of pixel intensities has the following form:

$D=\frac{1}{\sum_{i=1}^{N} I_{i}^{2}}$

$D$ shows the approximate number of filled lattice sites or pixels the distribution expands to. The spatial filling factor $(q)$ is the participation ratio divided by the total number of pixels $(N)$ :

$q=\frac{D}{N}$

The structural entropy $\left(S_{\text {str }}\right)$ is derived from the Shannon and Rényi entropies and was introduced by Pipek and Varga (1992) to study the structure of many electron density functions. For pixel intensity distributions the Rényi entropies (the generalization of the Shannon entropy) are defined in the following way:

$S_{n}=\frac{1}{1-n} \log \left(\sum_{i=1}^{N} I_{i}^{n}\right)$

It can be proven that the Shannon entropy is the first member of the Rényi entropies. The Shannon entropy measures how much the pixel intensity distribution $\left(I_{i}\right)$ deviates from the uniform distribution (when all $I_{i}$ have the same value, then the image is homogeneous gray). The second Rényi entropy is the entropy of a uniform distribution over $D$ sites (also called the extension part of the Shannon entropy) and corresponds to the number of pixels with significantly high intensity. The difference between $S_{1}$ and $S_{2}$ characterizes the structure of the system, and it is called the structural entropy $\left(S_{s t r}\right)$ :

$S_{s t r}=S_{1}-S_{2}=S-\log D$ 
The structural entropy characterizes purely the deviation of the intensity distribution from the step function (which is in fact the black and white image).

The pair of functions $\left(q ; S_{s t r}\right)$ of the distribution $\left\{I_{i}, i=1, \ldots, N\right\}$ is called generalized localization, and it can be used for analyzing the topology free structure of the observed distribution. Our postulated new parameter, the localization factor is the value $(\alpha)$ which yields the smallest square error between the generalized localization of the AFM image and the generalized localization of the $\exp \left(-x^{\alpha}\right)$ probe function. In other words, it can be defined as the minimum of the $E(\alpha)$ error function based on (14).

$E(\alpha)=\left[S_{\text {str }}(q)_{\text {image }}-S_{\text {str }}(q)_{\exp \left(-x^{\alpha}\right)}\right]^{2}$

In the case of the AFM images, the localization factor expresses the typical shape of hills and valleys of the topography maps. Two extremities of localization factor are $\alpha=0$ and $\alpha=\infty$ which correspond to totally flat surface and vertical-walled features, respectively.

To calculate the localization factor of an AFM image first the image is segmented to $K$ x $K$ number of smaller images. The pair of functions $\left(q ; S_{s t r}\right)$ will then be calculated for all of these segments, and $\alpha$ is determined as the minimum of the $E(\alpha)$ error function calculated for the $(q$; $\left.S_{s t r}\right)_{1 \ldots}\left(q ; S_{s t r}\right)_{\mathrm{K}}^{2}$ distribution and the generalized localization of the $\exp \left(-\mathrm{x}^{\alpha}\right)$ probe function (see Bonyár et al, 2012 for more details). This segmentation method grants useful properties to the localization factor. First, if the image contains local errors or artifacts (e.g. contaminations) they will only affect the local segment and not the whole image. For example the obtained image has two small dust particles which locally increase the measured height (seen as two bright spots on the image). By using a $6 x 6$ segmentation there will only be two segments which yield false $\left(q ; S_{s t r}\right)$ values (affected by the particles). Considering the whole 36 point $\left(q ; S_{s t r}\right)$ distribution, which form the generalized localization map and the $E(\alpha)$ error function, which is calculated for this distribution, their effect will be small. Secondly, the localization factor is less sensitive to the background correction of the obtained images, compared to the other frequently used parameters (such as surface roughness). This is due to the fact that the spatial frequency of the structures, which are to be characterized, is much higher than the piezo movement or the sample tilt which usually form the background noise. Inside a segment, the effect of low spatial frequency background is much smaller compared to the high frequency features of the structures. These claims were investigated and proved in our previous publication (Bonyár et al, 2013).

Although these benefits of the localization factor and segmentation - less sensitivity to noise, artifacts, contaminations, background correction etc. compared to average based parameters which are calculated for the whole image - are proved to be true, proper sample preparation, imaging and image post-processing is advised for optimal results. 


\subsection{Operation range and parameter optimization}

In order to obtain stable and meaningful localization factor values, the AFM image has to meet certain requirements. The parameters, which has an effect on the determinability of the localization factor are the scan size and the resolution of the image, and their relation to the lateral size of the structures, which are to be characterized. To explore the boundaries of this 'operation range' which is defined by these parameters a rather simplified model is used. The aim of this model and discussion is not to give optimal parameter sets for all of the different samples - such generalization would be impossible - but rather to illustrate and emphasize the effect of these parameters to help the users to find their optimal sets of parameters for their own samples to gain reliable and stable localization factor values.

Although in reality the structures has a variation in both size, shape and distribution, let us now consider a simplified model with a surface which has identical, hemispherical structures (e.g. grains) with a diameter of $d_{g}$, which are packed tightly together without overlapping. Creating an AFM image from this surface with a rectangular scan size of $A$ (where $A=l_{x} \times l_{y}$, and $l_{x}=l_{y}=$ l) and a resolution of $N$ (where $N$ is the total number of pixels and $N=M \times M$ ) defines the 'resolution' of a grain (which is other word the grain diameter in pixels, $M_{g}$ ) as in Eq. 15.

$M_{g}=\frac{M \times d_{g}}{l}$

To gain reliable localization factor values, a lower limit should be defined to $M_{g}$. In order to find this lower limit, the structural entropy $\left(S_{s t r}\right)$ and spatial filling factor $(q)$ values were calculated on hemispheres with different resolutions, as can be seen in Fig. 1. The relative values are calculated based on the values obtained on a hemisphere with a diameter of 100 pixel. It can be seen that above around 30 pixels the calculated $\left(q ; S_{s t r}\right)$ values stay inside the range of $\pm 5 \%$ of the end value, which means that further increasing the resolution does not yield more precise results.

Based on this we can say, that for every sample the size of the structures (e.g. grain size) should be measured and the image scan range and distribution should be set accordingly to ensure that every structure has proper resolution (at least $30 \mathrm{pixel} /$ diameter is advised).

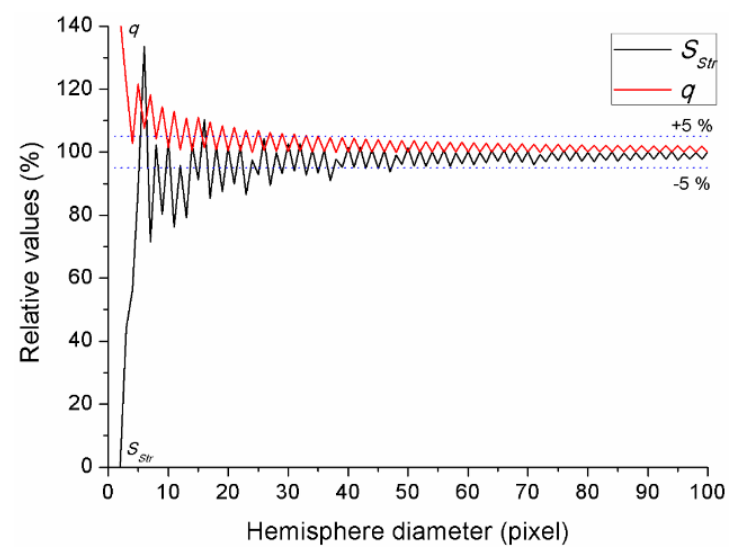

Fig.1. Relative values of the structural entropy $\left(S_{\text {str }}\right)$ and spatial filling factor $(q)$ values in function of the diameter of hemisphere structures, which they were calculated on. The relative values are calculated based on the values obtained on a hemisphere with a diameter of 100 pixel. 
The other limitation concerning the calculation of localization factor is connected to segmentation. As described in Section 2.2, the AFM image is first segmented into $K \times$ x $K$ pieces, the $\left(q ; S_{s t r}\right)$ values are calculated for every segment and the localization factor is determined based on these values as defined by Eq. 14. In one hand we need at least $3 \times 3$ segments (in this case the $E(\alpha)$ error function is determined on $9\left(q ; S_{s t r}\right)$ values, below this we can consider the result as unreliable), and generally the more segments are the better for the calculation of $E(\alpha)$. On the other hand, to prevent the loss of information we have to make sure that the segments of the image contain full structures and not only parts of it, so the value of $K$ is advised to fulfill Eq. (16), where the upper limit is determined according to the widely used Nyquist-Shannon sampling theorem from the field of signal processing.

$3 \leq K \leq \operatorname{round}\left(\frac{l}{2 d_{g}}\right)$

Overall, the operation range of the localization factor is determined by the complex relations between the scan size and resolution of the AFM image, the lateral size of the structures and the number of segments used for the calculation. Equations (15) and (16) describe these interdependencies and (with the recommendations of the author) can help the users optimize these parameters based on the properties of the samples. In Section 4.1 the whole procedure is illustrated for a specific application, which is the characterization of gold thin film microstructures.

\section{Materials and Methods}

\subsection{Materials}

Table 1 collects the technological parameters of the five different gold thin films which were used for the experiments. Thin films \#1-\#4 were used in the microstructure characterization experiments (Section 4.1), while thin film \#1 was used for the annealing experiments (Section 4.2).

Table 1. The technological parameters of the gold thin films used for the experiments

\begin{tabular}{|c|c|c|c|c|c|c|}
\hline ID & $\begin{array}{c}\text { Substrate } \\
\text { type }\end{array}$ & $\begin{array}{c}\text { Substrate } \\
\text { thickness [mm] }\end{array}$ & $\begin{array}{c}\text { Adhesion } \\
\text { layer type }\end{array}$ & $\begin{array}{c}\text { Adhesion layer } \\
\text { thickness [nm] }\end{array}$ & $\begin{array}{c}\text { Gold layer } \\
\text { thickness [nm] }\end{array}$ & Supplier \\
\hline \#1 & glass & 0.7 & $\mathrm{Ti}$ & $2 . .3$ & 350 & BUTE-FAT $^{*}$ \\
\hline \#2 & glass & 1.45 & $\mathrm{Ti}$ & 40 & 200 & $\begin{array}{c}\text { Optilab Kft. } \\
\text { (Hungary) }\end{array}$ \\
\hline \#3 & glass & 1 & $\mathrm{Cr}$ & 40 & 200 & MTA-MFA $^{* *}$ \\
\hline \#4 & polyester & 0.1 & $\mathrm{Ti}$ & 2.3 & 200 & BUTE-FAT $^{*}$ \\
\hline \#5 & glass & 1 & $\mathrm{Cr}$ & 5 & 220 & BUTE-FAT $^{*}$ \\
\hline
\end{tabular}

*BUTE-FAT: Budapest University of Technology and Economics (BUTE), Department of Atomic Physics (FAT)

**MTA-MFA: Hungarian Academy of Sciences (MTA), Research Institute for Technical Physics and Materials Science (MFA)

\subsection{AFM instrumentation}

AFM measurements were performed with a Veeco (lately Bruker) diInnova type scanning probe microscope (SPM). Contact-mode measurements were done with Bruker MSCT-AUNM-10 SiNi 
probes utilizing the longest tip with small spring constant $(k=0.01 \mathrm{~N} / \mathrm{m})$, while Bruker RTESPA$\mathrm{CP}$ probes were used for tapping-mode imaging (resonance frequency: $f_{0}=300 \mathrm{kHz}, k=40 \mathrm{~N} / \mathrm{m}$ ). The sampling rate of the images presented in the paper is either $512 \times 512$ or $1024 \times 1024$ obtained with $1 \mathrm{~Hz}$ scan rate. During the scans the feedback values of the proportional-integral-derivative (PID) controller were optimized according to the Veeco User Manual to gain the best image quality. The obtained images were post-processed with the Gwyddion 2.36 software (Nečas et al., 2012). Only standard background correction was applied on the images (to remove the piezo movement and sample tilt), and since all the investigated samples has small height/width ratio (the vertical size of the structures are small compared to the scan size) these corrections could easily be done without adding post-processing artifacts to the image.

\subsection{Other instrumentation}

For the thermal annealing experiments thin film \#5 was used with a Velp Scientifica ARE Heating Stirrer hot plate $\left(\max .300{ }^{\circ} \mathrm{C}\right.$ ). The temperature of the hot plate and the sample was measured with a Delta Ohm HD 2107.1 Pt thermometer. Two sets of annealing experiments were done referred to 'annealing1' (5 minutes at $\left.230{ }^{\circ} \mathrm{C}\right)$ and 'annealing2' ( 1 minute at $\left.280{ }^{\circ} \mathrm{C}\right)$ in the text.

For the investigation of the oxidation process at the Sn surface a SENJU Sn96.5Ag3.0Cu0.5 solder paste was printed onto a single $\mathrm{Cu}$ sheet and was soldered with hot air soldering. The sample were embedded in an acryl based (Technovit 4006) resin and then it was subjected to standard metallographic grinding/polishing. First it was ground by SiC grinding papers (grit size: 80, 320, $500,1200)$, then polished by $9 \mu \mathrm{m}, 3 \mu \mathrm{m}$ and $1 \mu \mathrm{m}$ and finally OP-S $(0.04 \mu \mathrm{m})$ diamond and $\mathrm{SiC}$ suspensions respectively.

Two sets of oxidation experiments were done, which will be referred to as 'ambient oxidation' and 'accelerated oxidation' in the future. First, AFM measurements were done on the freshly polished surface (as a reference), then the sample was placed into an ESPEC SH-241 HAST (highly accelerated stress test) chamber with the following conditions: relative humidity (RH) 85\%, temperature $85{ }^{\circ} \mathrm{C}$ for four hours to oxidize the Sn surface. In the case of 'ambient oxidation' the freshly polished sample was left in laboratory ambient conditions $\left(22^{\circ} \mathrm{C}, 44 \% \mathrm{RH}\right)$ for one week.

\section{Results and Discussion}

\subsection{Characterization of gold thin film microstructures}

In the previous publication, where the localization factor was originally introduced for AFM images, the same technologically (and so structurally) different gold thin films were used to illustrate its possible application to characterize the shape of the grains (Bonyár et al., 2012). In that paper however, the operation range of the localization factor (as discussed in Section 2.3) was not investigated and thus unintentionally, the parameter was calculated without optimizing the parameters which affect its proper value. For example it was also calculated on large scan areas (e.g. $100 \mu \mathrm{m}^{2}$ and above) laying far out from the operation range, which thus resulted in unreliable values and caused the localization factor to decrease by increasing the scan size (see Fig. 8 in Bonyár et al., 2012). 
The aim of this section in one hand is to give an example and detailed illustration how to determine the operation range of the localization factor and select the best possible parameters (scan conditions and segmentation) for its determination, and in the other hand, to demonstrate that by staying within this operation range one can obtain stable and reliable values which is only slightly depending on the scan size of the image.

Fig. 2 presents AFM topography images with $4 \mu \mathrm{m}^{2}$ scan size of the four investigated thin film surfaces. The identically same surfaces were characterized in our previous publication (Bonyár et al., 2012), where detailed values of the measured surface roughness $\left(S_{a}, S_{q}\right)$, and developed area ratio (also called roughness factor, $\left.S_{d r}\right)$ are given for seven different scan sizes $(1,4,9,36,100$, 400, $900 \mu \mathrm{m}^{2}$ ), together with sample AFM images with $1 \mu \mathrm{m}^{2}$ and $36 \mu^{2}$ scan sizes.
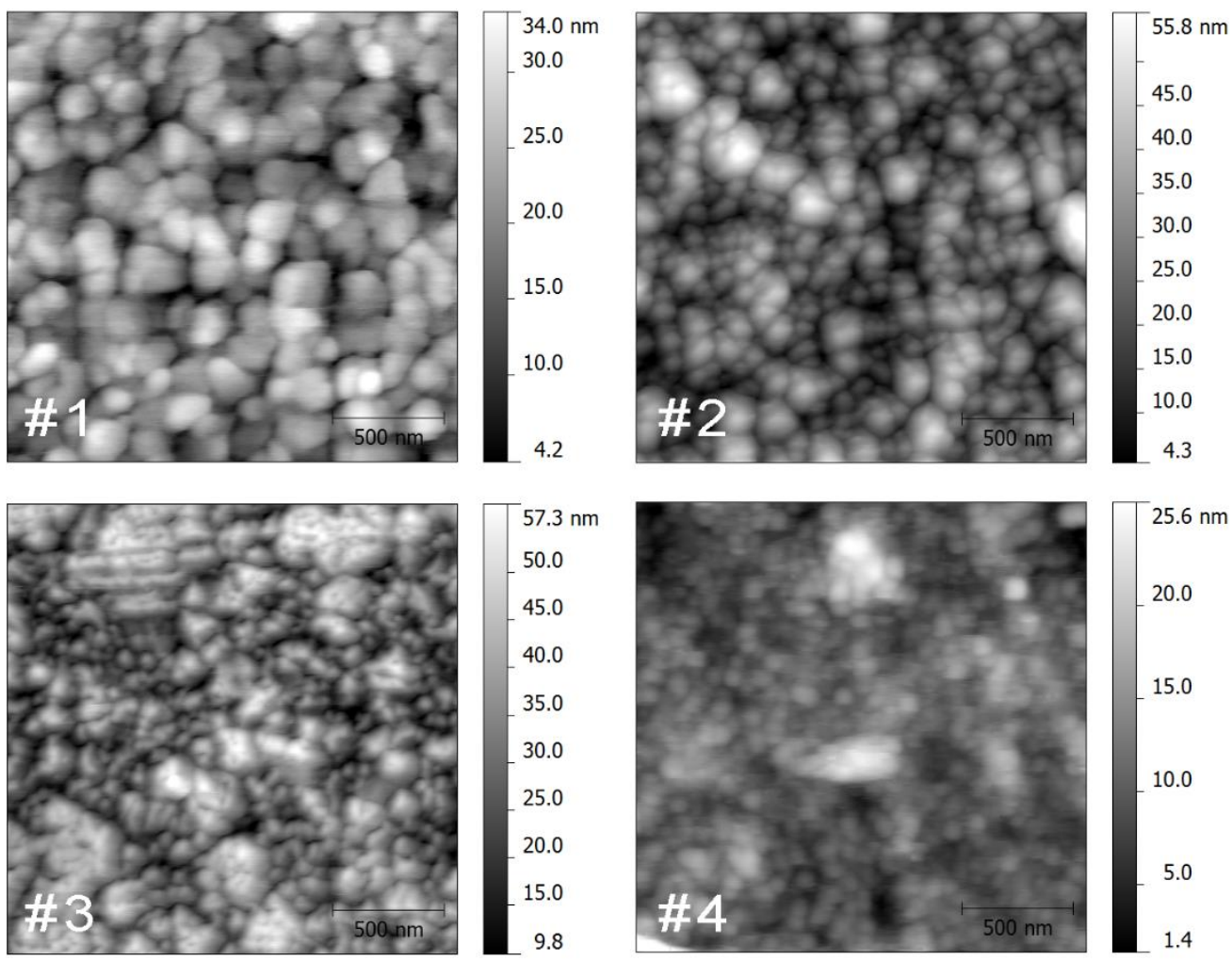

Fig.2. Contact-mode AFM images made on four different gold thin films (see Table 1 for the technological parameters. Scan size: $2 \times 2 \mu \mathrm{m}^{2}$.

In order to determine the operation range of the localization factor for these surfaces with the help of (15) and (16), first we need to know the size of the grains. Unlike in our proposed simple model, in the case of all realistic samples the grain size varies and has a distribution which is presented in Fig. 3 for the four thin films, which were measured on $1 \mu^{2}$ images. 


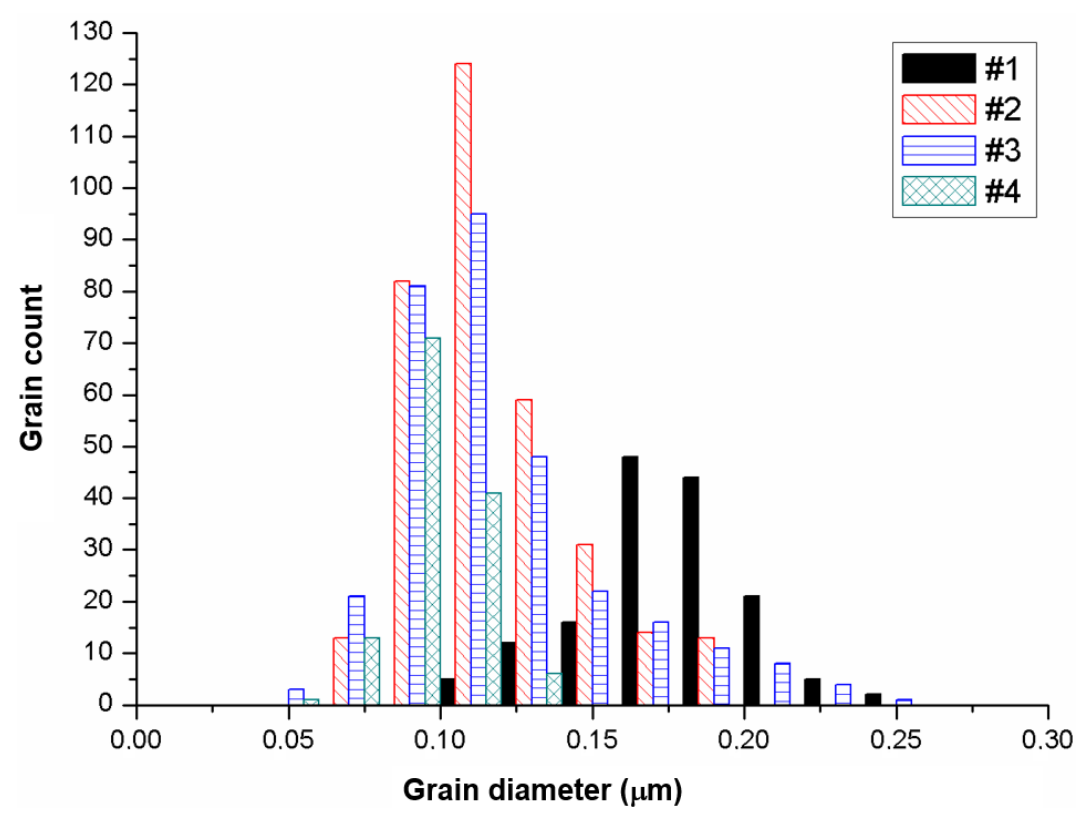

Fig.3. Grain diameter distributions measured on $1 \mu \mathrm{m}^{2}$ AFM images for the four investigated gold thin films.

In the light of the grain size distribution Fig. 4 can be drawn, which illustrates the recommended segment number ( $K$ x $K$ segmentation) calculated based on Eq. (16) for three rectangular scan sizes. To avoid losing information during segmentation, when selecting the maximum number of segments, one should always consider the largest grain size for one sample (right end of the bars in the top left corner of Fig. 4). Based on this we can conclude, that for samples \#1 and \#3 $1 \mu \mathrm{m}^{2}$ images cannot be used, only $4 \mu \mathrm{m}^{2}$ images with ( $\left.4 \mathrm{x} 4\right)$ segmentation and $9 \mu \mathrm{m}^{2}$ images with $(6 \mathrm{x}$ 6) segmentation.

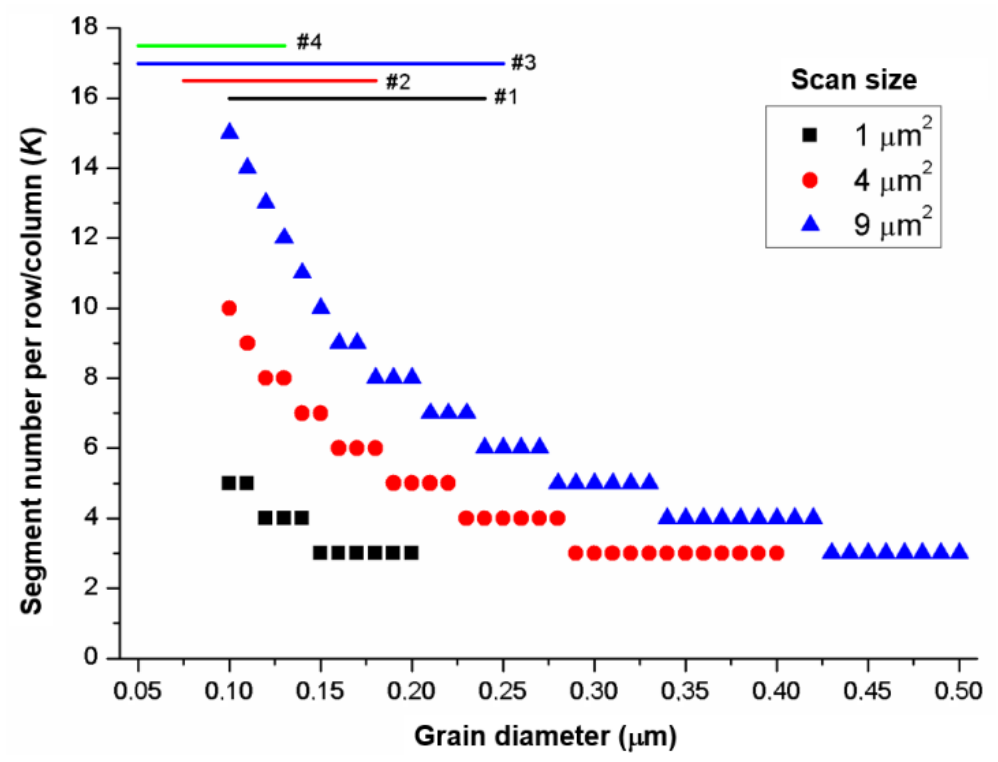

Fig.4. Segmentation recommendations based on the grain size distribution of the sample and the scan size of the image. The bars on the upper left corner represent the spread of the grain size distributions presented in Fig. 3. 
Grains with the smallest size (left side of the bars in the top left corner of Fig. 4) should also be taken into account regarding the resolution and scan size of the image. Based on Eq. (15), if $9 \mu \mathrm{m}^{2}$ images are obtained with a resolution of 1024 x 1024 (which is the highest resolution of the used AFM), then the smallest grains with a diameter of $50 \mathrm{~nm}$ will have an equivalent diameter of 17 pixels, which might cause a loss of information for these grains, based on Fig. 1. Considering that only a small percent of the grains are in this size range (see Fig. 3) $9 \mu^{2}$ images may be accepted as a borderline case, but no larger scan sizes are advised to be used.

Taking into account all of these considerations Table 2 collects the applied parameters for the final calculations. It can be seen in Fig. 5, that inside the operation range, with the optimized parameters, the localization factor gives stable values. However, in the case of sample \#4 the effect of decreased grain resolution can be seen in the small decrease at $9 \mu \mathrm{m}^{2}$. The values of the localization factor are in good accordance with what we can see on the AFM images: the higher value of thin film \#1 indicates that the grains have sharp features with steep walls and deep valleys (namely high wall contact angle), while the grains of thin films \#2 and \#4 are more like hills with gentle walls (smaller wall contact angle, smoother structures).

Table 2. The optimal scan size and segmentation values, which were used for the calculation of localization factor for the four different thin films.

\begin{tabular}{||c|c|c|c||}
\hline & \multicolumn{3}{|c||}{ Scan size } \\
\hline Thin film No. & $\mathbf{1} \boldsymbol{\mu m}^{\mathbf{2}}$ & $\mathbf{2} \boldsymbol{\mu \mathbf { m } ^ { 2 }}$ & $\mathbf{9} \boldsymbol{\mu \mathbf { m } ^ { 2 }}$ \\
\hline$\# 1$ & - & $4 \times 4$ & $7 \times 7$ \\
\hline$\# 2$ & $3 \times 3$ & $6 \times 6$ & $8 \times 8$ \\
\hline$\# 3$ & - & $4 \times 4$ & $6 \times 6$ \\
\hline$\# 4$ & $4 \times 4$ & $8 \times 8$ & $13 \times 13$ \\
\hline
\end{tabular}

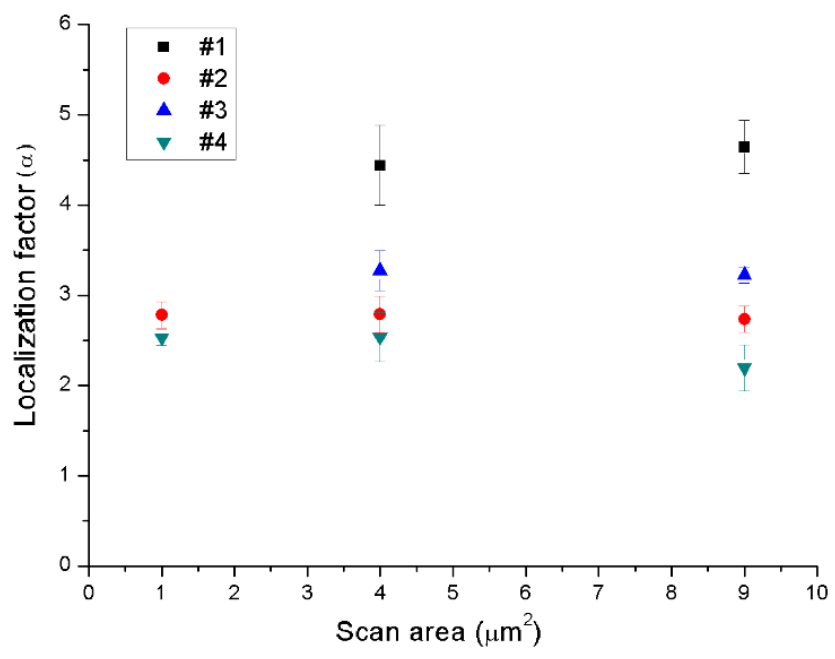

Fig.5. Localization factor $(\alpha)$ parameters calculated on the four investigated gold thin films as function of the scan area (an average of 5 images per area is presented). 


\subsection{Characterization of the effect of annealing on the microstructure of gold thin films}

In this section the effect of thermal annealing on the microstructure of gold thin films and the possibility to characterize this process with the localization factor parameter is investigated. It is known that by annealing it is possible to alter the microstructure of previously deposited thin films. The annealing temperature is often much lower than the melting point of the metal. In the case of gold - which has a melting point of $1064{ }^{\circ} \mathrm{C}$ - it is possible to modify the microstructure to a small extent even at temperatures as low as $200-300{ }^{\circ} \mathrm{C}$ in the order of minutes. By using higher temperatures and longer annealing times it is even possible to smooth out the surface and create nearly atomically flat terraces as demonstrated by Nogues et al. (2004) on MICA and Lauer et al. (2007) on glass substrates.

Since our goal here is to show that the changes in the grainy microstructure of the gold thin film can be characterized with the localization factor we only used low temperature annealing to modify the shape of the grains. Fig. 6 presents sample contact-mode topography images of the reference thin film surface before annealing (thin film \#5 was used for this purpose, see Table 1 for details) and after two short annealings at low temperature. Annealing 1 and 2 were not subsequent steps, in both cases the reference sample was annealed.
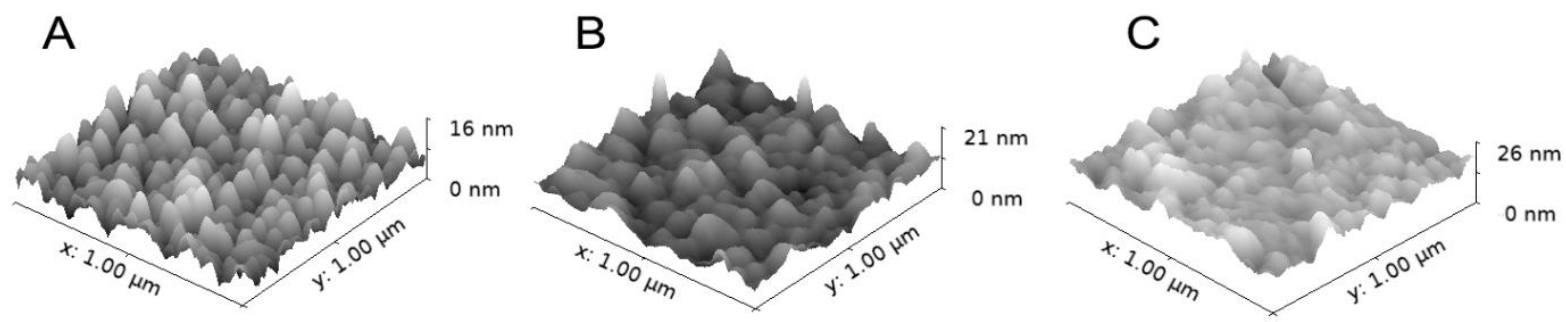

Fig.6. Contact-mode AFM topography images made on a gold thin film before and after annealing. A) reference, before annealing; B) after annealing1 $\left(5\right.$ minutes at $\left.230{ }^{\circ} \mathrm{C}\right)$; C) after annealing2 (1 minute at $\left.280{ }^{\circ} \mathrm{C}\right)$. Scan areas: 1 $\mu \mathrm{m}^{2}$.

It can be seen on Fig. 6 that the microstructure changes during the annealing, the grains are less defined, and for the first sight it seems that the surface is smoother and the grains are relaxed. However based on the numerical values presented in Table 3 we can see that the maximum height $\left(S_{z}\right)$ of the surface is significantly increased (36\% and $21 \%$ after annealing 1 and 2 respectively) and this is mostly due to the appearance of some high peaks on the surface (based on the values of $S_{p}$ ). This apparent paradox can also be seen by comparing the surface roughness $\left(S_{q}\right)$ and the developed area ratio $\left(S_{d r}\right)$ after annealing 2 . We expect the relaxation of residual stress and surface inhomogenities and thus the smoothening of the microstructure which is consistent with the $-20 \%$ decrease of $S_{d r}$, however in the same time the measured surface roughness shows an increase of 
16.7\%. This example clearly demonstrates and emphasizes the limitation of simple average based parameters like the surface roughness, since the appearance of a few high peaks can shift the measured roughness, while the majority of the grains undergo relaxation and smoothening.
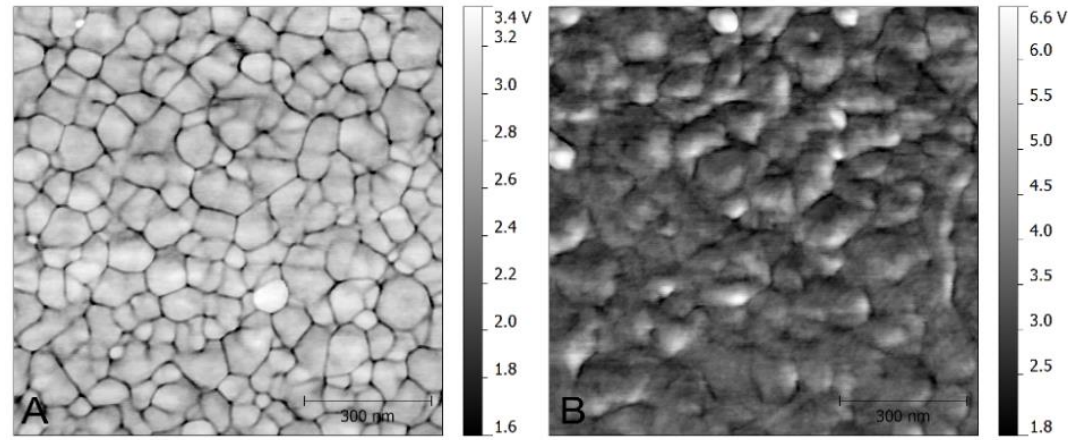

Fig.7. Tapping-mode AFM phase images of the gold thin film before annealing (left) and after annealing 1 for 5 minutes at $230{ }^{\circ} \mathrm{C}$ (right). Scan areas: $1 \mu \mathrm{m}^{2}$.

To prove this statement tapping-mode AFM images were made before and after annealing 1, as presented in Fig. 7. On these phase images the borders of the grains can clearly be seen, which boundaries start to disappear as the merging of the grains and elimination of the voids begin. This initial behavior of the grains is in good agreement with the work of Zhou et al. (2015), who recently reported similar changes in the grain structure (relaxation, void elimination at grain boundaries) after a long term annealing at lower temperature $\left(24 \mathrm{~h}\right.$ at $\left.60{ }^{\circ} \mathrm{C}\right)$. The results are also in good correspondence with the recent work of Arghir et al. (2016) who also reported a small increase in $S_{q}$ (around $9 \%$, from $2.2 \mathrm{~nm}$ to $2.4 \mathrm{~nm}$ ) after annealing a gold thin film for $7 \mathrm{~h} \mathrm{at} 180{ }^{\circ} \mathrm{C}$. The changes in the grain shape, the merging of the grains, and some scattered high peaks are also well observable in their presented topography images.

In the current experiments, the appearance of these high peaks after annealing 1 and 2 makes the precise determination of skewness $\left(S_{s k}\right)$ and kurtosis $\left(S_{k u}\right)$ nearly impossible, as can be seen in their high standard deviations (Table 3$)$. The increase in the autocorrelation length $\left(S_{a l}\right)$ also indicates that the inhomogeneity of the surface increased after annealing.

The obtained localization factor values are presented in Fig. 8 for two scan sizes (in Table 2 the scan size is $4 \mu \mathrm{m}^{2}$ ). After a small decrease for annealing 1 (around $-8 \%$ ) the localization factor shows a decrease of $-23 \%$ for annealing 2, which indicates the smoothening of the surface structures, a decreased steepness of the grain walls, as we see on the AFM images as well. We can say that with the localization factor it was possible to characterize the effect of the annealing process, while other parameters resulted in either inconsistent values or were impossible to determine in a reliable way. 


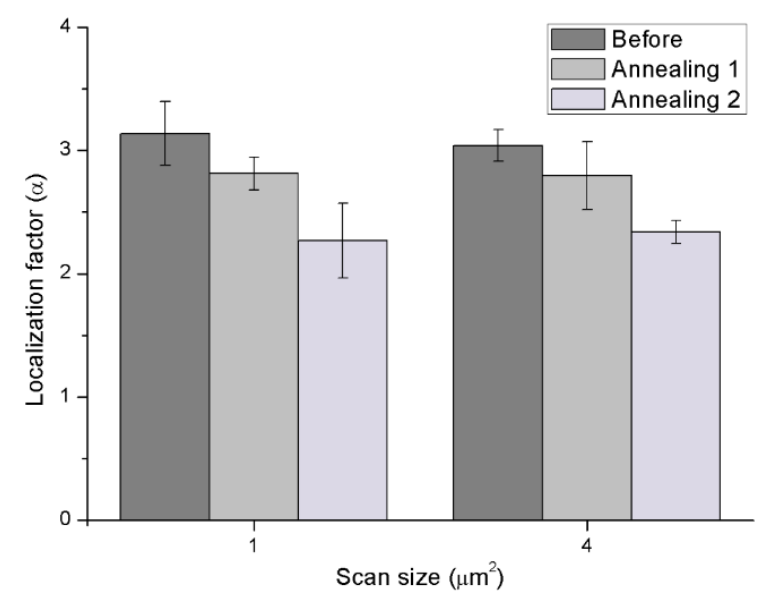

Fig.8. Localization factor values measured on the reference gold thin film (before annealing) and after annealing 1 (5 minutes at $\left.230{ }^{\circ} \mathrm{C}\right)$ and annealing $2\left(1\right.$ minute at $\left.280{ }^{\circ} \mathrm{C}\right)$. Images with scan sizes of $1 \mu \mathrm{m}^{2}$ and $4 \mu \mathrm{m}^{2}$ were used for the evaluation, a number of five images were evaluated in every case (resolution: 1024x1024).

\subsection{Detection of surface oxidation on Sn surface}

In this section the application of the localization factor parameter for the monitoring and characterization of the oxidation process on a polished tin surface is demonstrated. The oxidation of the tin surface prepared for metallographic inspection can occur under ambient laboratory conditions (Leidheiser, 1971) and its presence can sometimes be unfavorable since it can negatively affect subsequent sample preparation or investigation steps (e.g. it can mask against the electrochemical etching of tin, which is required for an electrochemical microstructure characterization method (Hurtony et al, 2015)). The formed oxide is transparent in the visible light region, so the optical microscopic inspection of the oxidation state is not possible. Since during the oxidation, the surface topology is also changing only to a small extent, the numerical characterization of this process with atomic force microscopy is also not trivial.

Fig. 9 presents sample topography images of the tin surface before and after oxidation. On the reference image made on the polished surface right after sample preparation (Fig 8/A) the effect of the polishing (scratches, holes) can clearly be seen. These scars represent local low areas on an otherwise flat surface, which is indicated by the measured negative skewness $(-0.56)$.

After 'ambient' oxidation (one week in laboratory ambient conditions, $22{ }^{\circ} \mathrm{C}, 44 \% \mathrm{RH}$, Fig. 9/B) the scars are less apparent, the sharp features left by polishing start to disappear, or at least soften (note that the smaller scratches are only barely visible in this phase). This could be explained by the expanding oxide which first rounds the sharp edges left after polishing, then starts to fill the trenches and holes. Numerically this is indicated by a small decrease in the measured surface roughness $(-13.84 \%)$, a decrease in the absolute value of skewness $(-61.8 \%)$, and a significant decrease in the developed area ratio $(-68.17 \%)$ (see Table 2 for details).

Fig. 9/C shows the effect of 'accelerated' oxidation $\left(2 \mathrm{~h}\right.$ in a HAST chamber at $85{ }^{\circ} \mathrm{C}$ and 85 $\% \mathrm{RH})$. The surface structure changed significantly: grainy tin oxide covers the surface. The grains fill the remaining trenches and are distributed evenly on the surface. This homogeneous distribution is indicated by the higher texture aspect ratio $\left(S_{t r}=0.65\right)$ and the balanced height distribution 
marked by the small positive skewness $\left(S_{s k}=0.14\right)$. The surface roughness $\left(S_{q}\right)$ is only $4.78 \%$ smaller compared to the reference, but the developed area ratio $\left(S_{d r}\right)$ is increased with $15.22 \%$ due to the oxide grain formation
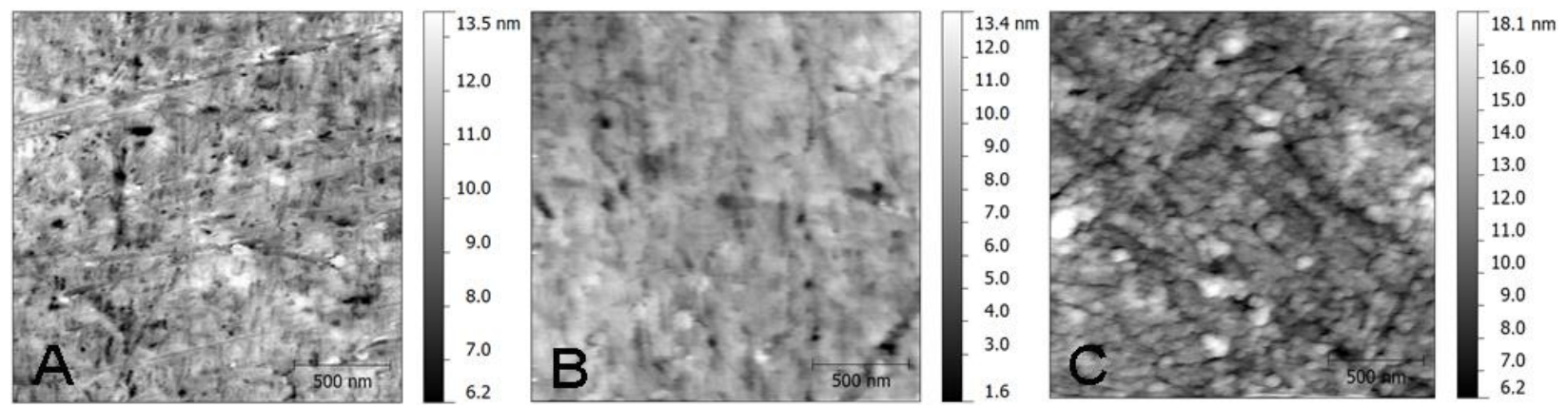

Fig.9. Contact-mode AFM topography images made on A) a polished tin surface; B) after 'ambient oxidation' (one week in laboratory ambient conditions, $\left.22{ }^{\circ} \mathrm{C}, 44 \% \mathrm{RH}\right)$; C) after 'accelerated oxidation' ( $2 \mathrm{~h}$ at $85{ }^{\circ} \mathrm{C}$ and $85 \%$ $\mathrm{RH})$.

The localization factor values which were measured on the polished and oxidized samples are presented in Fig. 10. The sharp features of the polished sample resulted in a high average localization factor (6.5), which correlates well with the observed large steps in height at these local areas of defects (holes, scratches). The softening of the features after 'ambient' oxidation resulted in a $-24.31 \%$ decrease in the localization factor, although the SD of its determination increased. The high SD of the localization factor indicates an inhomogeneous surface, which is true for our samples. After 'accelerated' oxidation the localization factor showed a total decrease of $-38.72 \%$ compared to the reference. This corresponds well with the softening of the features and the formation of the oxide grains. The obtained $\alpha=3.98$ for the grainy surface also corresponds well with the previous results, since the localization factor values ranged between $2.5-4.2$ for polycrystalline gold thin film grains.

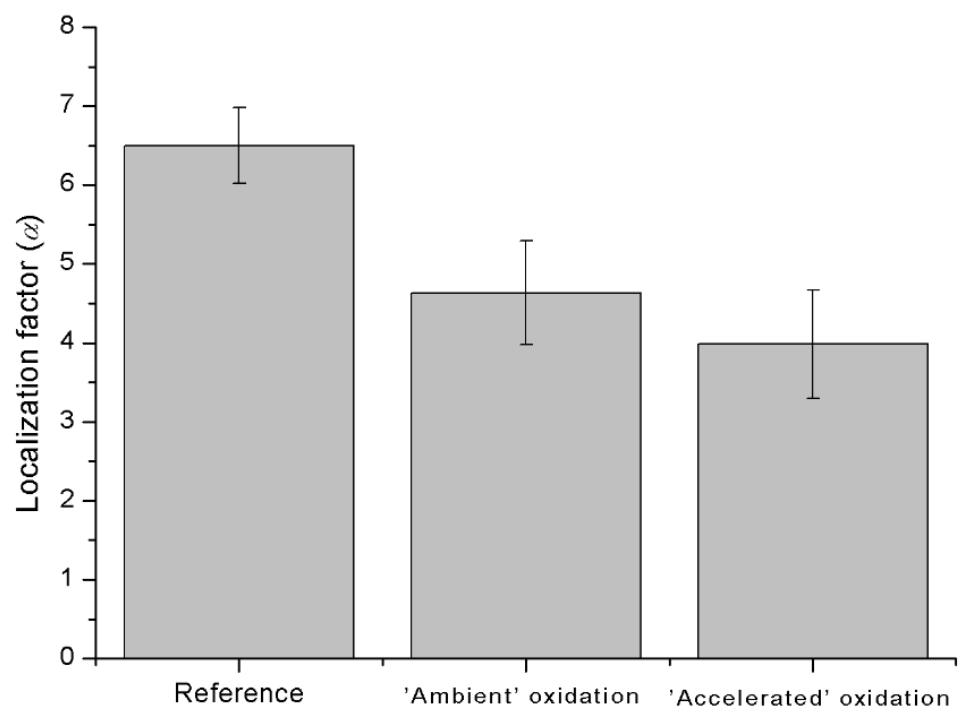

Fig.10. Localization factor values measured on the polished reference tin surface, after 'ambient' oxidation and after 'accelerated' oxidation. A number of six images with a scan size of $4 \mu \mathrm{m}^{2}$ were used in every case. 


\section{Conclusions}

Three examples were presented to demonstrate, that the localization factor can be utilized to characterize the shape of the surface structures, as long as the scanning and calculation parameters are set in a way to fulfill the recommended operation range conditions for its determination. The localization factor successfully characterized processes which altered the shape/features on the surface. Both the relaxation of the grainy structure of gold thin films during annealing and the softening of a polished tin surface followed by subsequent oxide grain formation during oxidation could be well characterized with this parameter. The measured changes in the localization factor was found to be consistent with the changes of other parameters which carry information regarding the structural changes (surface roughness, skewness, developed area ratio), but only if these parameters could reliably be determined.

\section{Acknowledgements}

This paper was supported by the János Bolyai Research Scholarship of the Hungarian Academy of Sciences.

\section{References}

Arghir, I. A., Schouteden, K., Goos, P., Delport, F., Spasic, D., Lammertyn, J., 2016. Thermal annealing of gold coated fiber optic surfaces for improved plasmonic biosensing. Sensors and Actuators B: Chemical $229,678-685$.

Bell, R.J., Dean, P., 1970. Atomic vibrations in vitreous silica. Discuss. Faraday Soc., 50-55.

Bonyár, A., Molnár, L. M., Harsányi, G., 2012. Localization factor: a new parameter for the quantitative characterization of surface structure with atomic force microscopy (AFM). Micron 43, 305-310.

Bonyár, A., Molnár, L.M., Harsányi, G., 2013. Dependence of the surface roughness and localization factor parameters on the background correction of AFM images: a thin film characterization study. Materials Science Forum 729, 193-198.

Chen, Y., Xuan, Y., 2015. The influence of surface roughness on nanoscale radiative heat flux between two objects. Journal of Quantitative Spectroscopy and Radiative Transfer 158, 52-60.

Hansson, S., Loberg, J., Mattisson, I., Ahlberg, E., 2011. Global biomechanical model for dental implants. J. Biomech. 44, 1059-1065.

Hurtony, T., Gordon, P., 2015. The correlation between the mechanical and electrochemical properties of solder joints, Proc. Of the 38th IEEE-ISSE International Spring Seminar on Electronics Technology, 70-74, DOI: $10.1109 /$ ISSE.2015.7247965

Lauer, M. E., Jungmann, R., Kindt, J.H., Magonov, S., Fuhrhop, J.H., Oroudjev, E., Hansma, H.G., 2007. Formation and healing of micrometer-sized channel networks on highly mobile Au(111) surfaces. Langmuir $23,5459-5465$.

Lee, G-J., Park, K-H., Park, Y-G., Park, H-K., 2010. A quantitative AFM analysis of nano-scale surface roughness in various orthodontic brackets. Micron 41, 775-782.

Leidheiser, H., 1971. The Corrosion of Copper, Tin and their Alloys. Wiley, NewYork 
Molnár, L. M., Nagy, Sz., Mojzes, I., 2010. Structural entropy in detecting background patterns of AFM images. Vacuum 84, 179-183.

Mukherjee, R., Chaudhury, K., Das, S., Sengupta, S., Biswas, P., 2012. Posterior capsular opacification and intraocular lens surface micro-roughness characteristics: An atomic force microscopy study. Micron 43, 937-947.

Nečas, D., Klapetek, P., 2012. Gwyddion: an open-source software for SPM data analysis, Cent. Eur. J. Phys. 10(1) 181-188.

Nečas, D., Klapetek, P., 2013. One-dimensional autocorrelation and power spectrum density functions of irregular regions. Ultramicroscopy 124, 13-19.

Nogues, C., Wanunu, M., 2004. A rapid approach to reproducible, atomically flat gold films on mica. Surface Science 573, L383-L389.

Pipek, J., 1989. Localization measure and maximum delocalization in molecular systems. Int. J. Quantum Chem. 36, 487-501.

Pipek, J., Varga, I., 1992. Universal classification scheme for the spatial-localization properties of oneparticle states in finite, $d$-dimensional system. Phys Rev A. 46, 3148-3163.

Rahaman, M.L., Zhang, L., Liu, M., Liu, W., 2015. Surface roughness effect on the friction and wear of bulk metallic glasses. Wear 332-333, 1231-1237.

Raposo, M., Ferreira, Q., Ribeiro, P.A., 2007. A Guide for Atomic Force Microscopy Analysis of SoftCondensed Matter. Formatex, Spain, 758- 769.

Wang, Y., Song, Z., Xu, Z., 2015. Mechanistic transition of heat conduction in two-dimensional solids: A study of silica bilayers. Phys. Rev. B 92, 245427.

Xia, W., Ni, C., Xie, G., 2016. The influence of surface roughness on wettability of natural/gold-coated ultra-low ash coal particles. Powder Technology 288, 286-290.

Zhou, S., Ji, Z., Shao, T., 2015. Residual stress evolution during long-term and cyclic aging and annealing of gold films deposited by electron beam evaporation. Vacuum 120, 132-138.

\section{Captions}

Fig.1. Relative values of the structural entropy $\left(S_{s t r}\right)$ and spatial filling factor $(q)$ values in function of the diameter of semisphere structures, which they were calculated on. The relative values are calculated based on the values obtained on a hemisphere with a diameter of 100 pixel.

Fig.2. Contact-mode AFM images made on four different gold thin films (see Table 1 for the technological parameters. Scan size: $2 \times 2 \mu \mathrm{m}^{2}$.

Fig.3. Grain diameter distributions measured on $1 \mu \mathrm{m}^{2}$ AFM images for the four investigated gold thin films.

Fig.4. Segmentation recommendations based on the grain size distribution of the sample and the scan size of the image. The bars on the upper left corner represent the spread of the grain size distributions presented in Fig. 3.

Fig.5. Localization factor $(\alpha)$ parameters calculated on the four investigated gold thin films as function of the scan area (an average of 5 images per area is presented). 
Fig.6. Contact-mode AFM topography images made on a gold thin film before and after annealing. A) reference, before annealing; B) after annealing1 (5 minutes at $\left.\left.230{ }^{\circ} \mathrm{C}\right) ; \mathrm{C}\right)$ after annealing2 $(1$ minute at 280 $\left.{ }^{\circ} \mathrm{C}\right)$. Scan areas: $1 \mu \mathrm{m}^{2}$.

Fig.7. Tapping-mode AFM phase images of the gold thin film before annealing (left) and after annealing1 for 5 minutes at $230{ }^{\circ} \mathrm{C}$ (right). Scan areas: $1 \mu \mathrm{m}^{2}$.

Fig.8. Localization factor values measured on the reference gold thin film (before annealing) and after annealing $1\left(5\right.$ minutes at $\left.230{ }^{\circ} \mathrm{C}\right)$ and annealing $2\left(1\right.$ minute at $\left.280{ }^{\circ} \mathrm{C}\right)$. Images with scan sizes of $1 \mu \mathrm{m}^{2}$ and $4 \mu \mathrm{m}^{2}$ were used for the evaluation, a number of five images were evaluated in every case (resolution: 1024x1024).

Fig.9. Contact-mode AFM topography images made on A) a polished tin surface; B) after 'ambient oxidation' (one week in laboratory ambient conditions, $22{ }^{\circ} \mathrm{C}, 44 \% \mathrm{RH}$ ); C) after 'accelerated oxidation' $\left(2 \mathrm{~h}\right.$ at $85^{\circ} \mathrm{C}$ and $\left.85 \% \mathrm{RH}\right)$.

Fig.10. Localization factor values measured on the polished reference tin surface, after 'ambient' oxidation and after 'accelerated' oxidation. A number of six images with a scan size of $4 \mu \mathrm{m}^{2}$ were analized in every case.

Table 1. The technological parameters of the gold thin films used for the experiments

Table 2. The optimal scan size and segmentation values, which were used for the calculation of localization factor for the four different thin films.

Table 3. The relevant 3D areal parameters and the calculated localization factors $(\alpha)$ measured in the gold thin film annealing and in the tin oxidation experiments. An average of at least 5 image is presented in every case with standard deviations. $\Delta$ indicates the relative change in that parameter compared to the reference value. All values were calculated on $4 \mu \mathrm{m}^{2}$ images. 